\title{
Culture of Pig Induced Pluripotent Stem Cells without Direct Feeder Contact in Serum Free Media
}

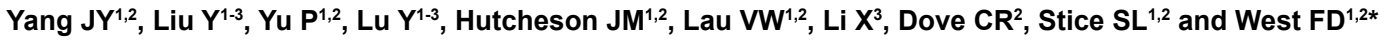 \\ ${ }^{1}$ Regenerative Bioscience Center, University of Georgia, Athens, GA 30602, USA \\ ${ }^{2}$ Department of Animal and Dairy Science, University of Georgia, Athens, GA 30602, USA \\ ${ }^{3}$ State Key Laboratory for Conservation and Utilization of Subtropical Agro-bioresources, Animal Reproduction Institute, Guangxi University, Nanning, Guangxi 530004, \\ China
}

\begin{abstract}
Background: Reprogramming pig somatic cells into induced pluripotent stem cells (iPSCs) have promising applications in basic biology, disease model development and xenotransplantation. In the mouse, embryonic stem cell (ESC) technology has revolutionized the field enabling gene targeting, complex screening strategies and the creation of animals that show unique characteristics of interest. Recent breakthroughs utilizing induced pluripotent stem cell technology in the pig have made it possible to produce pig pluripotent stem cells that resemble germline chimeric competent mouse ESCs. However, an optimal culture system for piPSC expansion has yet to be developed. Most reports have maintained piPSCs in undefined systems that use xenoproducts and feeder layers, which are potential sources of contamination.
\end{abstract}

Methods: In this study, new lines of pig iPSCs (piPSC) were generated from pig fibroblast cells by overexpressing six reprogramming genes: POU5F1, SOX2, NANOG, LIN28, KLF4 and C-MYC. These new lines were tested for their ability to be maintained on a Matrigel substrate in the established mouse 2i+LIF system, the human mTeSR1 system and variations of a feeder conditioned media system. Analysis and identification of piPSCs were performed using immunocytochemistry, flow cytometry and by examining embryoid body formation and differentiation.

Results: The newly generated piPSCs showed morphological features, immunoreactivity and reactivation of endogenous pluripotency networks consistent with iPSCs. Similar to cells cultured on feeders, piPSCs maintained under all 7 feeder-free conditions expressed POU5F1 and NANOG, SSEA-1, SSEA-4 and TRA1-81. However, flow cytometry demonstrated that piPSCs cultured in feeder conditioned media with KnockOut Serum Replacement and basic fibroblast growth factor (FGF2) showed significantly higher levels of SSEA1 and SSEA4 expression than cells cultured in a 2i+LIF or mTeSR1 system.

Conclusion: These findings demonstrate that piPSCs can be maintained in defined systems without serum and direct feeder contact, increasing their potential use in both agricultural and biomedical fields.

Keywords: Culture system; iPSC; Pig; Reprogramming; Stem cells; Pluripotency; Feeder free

Abbreviations: AP: Alkaline Phosphatase; FGF2: Basic Fibroblast Growth Factors; ERK: Extracellular Signal-regulated Kinase; ESC: Embryonic Stem Cell; GSK3: Glycogen Synthase Kinase 3; iPSC: induced Pluripotent Stem Cells; KSR: Knockout Serum Replacement; LIF: Leukemia Inhibitory Factor; MEK: Mitogen-activated Protein Kinase; SCNT: Somatic Cell Nuclear Transfer

\section{Introduction}

The pig is an important species as a food source in animal agriculture and as a large animal disease and injury model in the biomedical arena [1-4]. To further the agricultural and biomedical utility of pigs, great efforts have been made to genetically manipulate these animals through the development of technologies such as somatic cell nuclear transfer (SCNT) $[2,5,6]$. However, the ability to generate transgenic pigs with extensive genetic modifications (e.g. multiple gene knock in and/or knock outs) using SCNT is limited. In the mouse, embryonic stem cell (ESC) technology has revolutionized the field by enabling gene targeting, complex screening strategies (e.g. ENU screening) and by allowing the creation of animals that show unique characteristics of interest [7-12]. The ability to do this in other species has been limited by the inability to derive pluripotent stem cells that can be manipulated and used to generate germline competent chimeric animals [13-15]. In the pig, recent breakthroughs utilizing induced pluripotent stem cell
(iPSC) technology have made it possible to produce pig pluripotent stem cells that resemble germline chimeric competent mouse ESCs [16-19].

Pig iPSCs (piPSCs) have recently been generated by overexpression of different combinations of the reprogramming genes POU5F1, SOX2, NANOG, LIN28, KLF4 and C-MYC [16,20-23]. These cells show typical pluripotent stem cell morphology and express pluripotent genes and proteins such as alkaline phosphatase (AP), SSEA1 and SSEA4. Importantly, these cells have also shown significant plasticity in vitro and in vivo by forming cells of all three germ layers in embryoid bodies and teratomas. piPSCs have now been demonstrated to contribute with high efficiency to the germlines of chimeric animals allowing for the production of transgenic offspring [16,17]. These data

*Corresponding author: Franklin D West, 425 River Road, ADS, University of Georgia, Athens, GA 30602, USA, Tel: 706-542-0988; Fax: 706-542-7925; E-mail: westf@uga.edu

Received January 16, 2014; Accepted February 20, 2014; Published February 22, 2014

Citation: Yang JY, Liu Y, Yu P, Lu Y, Hutcheson JM, et al. (2014) Culture of Pig Induced Pluripotent Stem Cells without Direct Feeder Contact in Serum Free Media. J Stem Cell Res Ther 4: 174. doi:10.4172/2157-7633.1000174

Copyright: ( 2014 Yang JY, et al. This is an open-access article distributed under the terms of the Creative Commons Attribution License, which permits unrestricted use, distribution, and reproduction in any medium, provided the original author and source are credited. 
demonstrate that bona fide pluripotent stem cells can be generated from the pig, opening the possibility for genetic manipulations similar to those achieved in rodent models. Despite these advances, an optimal culture system for piPSC expansion has yet to be developed. piPSCs have been traditionally derived and maintained on feeder cells in media containing serum (e.g. fetal bovine serum, fetal calf serum) [17,20-22]. The maintenance of piPSCs under more defined conditions would be preferable as variability of serum and feeder cell preparations have been linked to uncontrolled and insidious alterations in pluripotency, differentiation potential and cellular growth patterns [24]. In the context of xenotransplantation, feeder cells and xeno products act as potential sources of viral and prion contamination and increase the concern for graft rejection by increasing the immunoreactivity of cells [25]. Current systems to expand human iPSCs are more defined, utilizing extracellular matrices such as Matrigel over feeder cells and using media types such as mTeSR1. In the mouse ESC $2 \mathrm{i}+\mathrm{LIF}$ system, culture media has been supplemented with small molecules that alleviate differentiation cues and stabilize signaling pathways that maintain pluripotency [26]. PD0325901 and CHIR99021 are two small molecules that inhibit mitogen-activated protein kinase (MEK1/2) and glycogen synthase kinase 3 (GSK3) respectively. LIF is a key signaling factor that activates the JAK-STAT pathway, the self-renewal pathway in pluripotent stem cells. These factors maintain mouse ESCs and iPSCs in a naive state, a pluripotent state that is more capable of contributing to germline chimeric animals [26,27].

In this study, we derive two piPSC lines that show morphological features of iPSCs and express a number of the key stem cell markers including SSEA1, SSEA4 and TRA-1-81. piPSCs showed similar expansion patterns and proliferation rates as hESCs and were able to maintain karyotypic stability in extended culture. We then examined the ability of seven feeder free culture systems to maintain these cells in a pluripotent state based on SSEA1 and SSEA4 expression.

\section{Material and Methods}

\section{Cell lines, culture and transduction}

Pig dermal fibroblasts were derived from a skin biopsy of a Yorkshire cross pig. Fibroblasts were expanded and maintained in fibroblast growth medium consisting of Dulbecco's modified Eagle medium (DMEM) high glucose (Hyclone, UT, USA), $10 \%$ fetal bovine serum (FBS; Hyclone, UT, USA), $4 \mathrm{mM}$ L-Glutamine (Gibco, NY, USA), $50 \mathrm{U} / \mathrm{ml}$ penicillin (Gibco, NY, USA) and $50 \mu \mathrm{g} /$ $\mathrm{ml}$ streptomycin (Gibco, NY, USA). Cells were maintained in $5 \%$ $\mathrm{CO} 2$ at $37^{\circ} \mathrm{C}$. For transduction, a total of 120,000 pig fibroblast cells were plated in one well of a 4 -well plate. After $24 \mathrm{hrs}$, pig fibroblast cells underwent lentiviral transduction utilizing a viPS kit (Thermo Scientific, UT, USA) with viruses containing the human stem cell genes POU5F1, NANOG, SOX2, LIN28, KLF4 and C-MYC under the promoter of human elongation factor-1 alpha (EF1- $\alpha)$. Transduction was performed using 1X TransDux (System Biosciences, CA, USA). Pig fibroblast cells were trypsinized $24 \mathrm{hrs}$ after transduction and passaged onto inactivated mouse embryonic fibroblast feeder cells in embryonic stem cell expansion medium DMEM/F12 (Gibco, NY, USA) supplemented with $20 \%$ Knockout Serum Replacement (KSR; Gibco, NY, USA), $2 \mathrm{mM}$ L-glutamine (Gibco, NY, USA), $0.1 \mathrm{mM}$ non-essential amino acids (Gibco, NY, USA), $50 \mathrm{U} / \mathrm{ml}$ penicillin (Gibco, NY, USA), $50 \mu \mathrm{g} / \mathrm{ml}$ streptomycin (Gibco, NY, USA), 0.1 $\mathrm{mM} \beta$-mercaptoethanol (Sigma-Aldrich, MO, USA) and $10 \mathrm{ng} / \mathrm{ml}$ FGF2 (Sigma-Aldrich, MO, USA and R\&D Systems, MN, USA). Pig
iPSC colonies were manually harvested and plated on Matrigel (BD Biosciences, MA, USA; diluted 1:100 in DMEM/F12) coated dishes in seven different media: 1. mTeSR1: mTeSR1 (Stemcell Technologies, Vancouver, Canada); 2. KSRF: conditioned (media exposed to feeder cells for 24 hours) KSR (cKSR) $+10 \mathrm{ng} / \mathrm{ml} \mathrm{FGF2;} \mathrm{3.} \mathrm{KSRFL:} \mathrm{cKSR+10}$ ng/ml FGF2+10 ng/ml LIF (EMD Millipore, MA, USA); 4. KFC: cKSR+10 ng/ml FGF2+3 uM CHIR99021 (EMD Millipore, MA, USA); 5. KFP: cKSR+10 ng/ml FGF2+0.8 uM PD0325901 (Sigma-Aldrich, MO, USA); 6 . KFCP: cKSR+10 ng/ml FGF2+3 uM CHIR99021+0.8 uM PD0325901; 7. NCP: N2B27 (Life Technologies, NY, USA)+3 uM CHIR99021+0.8 uM PD0325901.

Pig iPSCs were passaged using dispase $(1 \mathrm{mg} / \mathrm{ml}$; Life Technologies, NY, USA) every 3 to 4 days. Karyotype analysis was performed after 20 passages under feeder-free conditions by a standard high-resolution G-banding method at Cell Line Genetics (WI, USA). The hESC line, WA09 (H9) (46, XX karyotype), was purchased from WiCell Research Institute (WI, USA). The cervical adenocarcinoma cell line, HeLa was purchased from ATCC (VA, USA). The IMR-90 human lung fibroblast derived hiPSC (46, XX karyotype) was a gift from ArunA Biomedical, Inc (GA, USA).

\section{Alkaline phosphatase, immunocytochemistry and flow cytometry}

AP staining was carried out with the VECTOR Red Alkaline Phosphatase Substrate Kit (Vector Laboratories, CA, USA) according to the manufacturer's instructions.

The immunostaining protocol used was previously reported [28]. Briefly, cells were washed with PBS+/+ (Thermo Scientific, UT, USA) and fixed with $4 \%$ paraformaldehyde (Sigma-Aldrich, MO, USA) at room temperature for 15 minutes. For intracellular staining, cells were permeabilized with $0.1 \%$ Triton X-100 (Sigma-Aldrich, MO, USA) and $1 \%$ polyvinylpyrrolidone (PVP; Sigma-Aldrich, MO, USA) in a PBS blocking solution containing $4 \%$ normal fetal bovine serum. For extracellular staining, cells were blocked in PBS containing 4\% normal fetal bovine serum. Primary antibodies used were POU5F1 (R\&D Systems, MN, USA, 1:200), SOX2 (R\&D Systems, MN, USA, 1:200), NANOG (Millipore, MA, USA, 1:200), BIII-TUBULIN (Neuromics, MN, USA, 1:200), aSMA (Santa Cruz, CA, USA, 1:100), Vimentin (R\&D Systems, MN, USA, 1:200), SSEA1 (Developmental Studies Hybridoma Bank, IA, USA, 1:200), SSEA4 (Developmental Studies Hybridoma Bank, IA, USA, 1:200), TRA-1-60 (Millipore, MA, USA, 1:200) and TRA-1-81 (Millipore, MA, USA, 1:200). Primary antibodies were detected using fluorescently conjugated secondary antibodies Alexa Fluor 488 (Life Technologies, NY, USA, 1:500) and 594 (Life Technologies, NY, USA, 1:500). Cell observations and images were captured on an Ix81 with Disc-Spinning Unit (Olympus, NY, USA) using Slide Book Software (Intelligent Imaging Innovations).

For flow cytometry, cells were fixed with $4 \%$ paraformaldehyde for 15 minutes. Cells were blocked in $4 \%$ horse serum (Sigma-Aldrich, MO, USA) for 45 minutes. Primary antibodies were directed against SSEA1 (1:200) and SSEA4 (1:200). Primary antibodies were detected using fluorescently conjugated secondary antibody Alexa Fluor 488 (1:500). Cells were analyzed using a Dakocytomation Cyan (Beckman Coulter, FL, USA) and FlowJo Cytometry analysis software (Tree Star, Inc, OR, USA).

\section{Proliferation and telomerase activity}

The proliferation assay was performed by manual counts $(n=3)$ 
at $12,24,36$ and $48 \mathrm{hrs}$ after plating. Population doubling time was determined using an exponential regression curve fitting approach (http://www.doubling-time.com/compute.php). Telomerase activity of pig fibroblast cells, pig iPSCs, WA09 and HeLa cells (positive control) was determined using the TRAPeze XL Telomerase Detection Kit (Millipore, MA, USA) following the manufacturer's instructions. Telomerase levels were reported in units of total product generated (TPG). Statistical analysis was performed utilizing ANOVA and Tukey pair-wise comparisons between each population with a $\mathrm{p}$-value $<0.05$ being considered significant.

\section{Embryoid body formation and differentiation}

Embryoid bodies (EBs) were formed by plating $2.0 \times 106$ pig iPSCs in mTeSR1 medium and $0.1 \mathrm{mM} \mathrm{Y-27632} \mathrm{ROCK} \mathrm{inhibitor} \mathrm{(Stemgent,}$ CA, USA) in an AggreWell plate (Stemcell Technologies, Vancouver, Canada). After $24 \mathrm{hrs}$, aggregates were harvested and maintained in $20 \%$ KSR media without FGF2 for 8 days. To assess differentiation through immunocytochemistry, EBs were plated on 4-well chamber slides (BD Biosciences, MA, USA) and maintained in 20\% KSR media without FGF2, which allowed for further differentiation over 2 additional days.

\section{RNA isolation, cDNA preparation/synthesis and RT-PCR}

RNA was isolated using the RNeasy QIAprep Spin miniprep Kit (Qiagen, CA, USA) per the manufacturer's instructions. Genomic DNA was removed using gDNA eliminator columns (Qiagen, CA, USA). RNA quality and quantity were determined using the NanoDrop 8000 (Thermo Scientific, UT, USA). Total mRNA (500ng) extractions were reverse transcribed into cDNA using iScript cDNA Synthesis Kit (Bio-Rad Laboratories, CA, USA). PCR amplification was performed using GoTaq Green master mix (Promega, WI, USA). Primers used in RT-PCR are listed in Table 1. PCR reactions were performed by initially denaturing cDNA at $95^{\circ} \mathrm{C}$ for 3 min followed by 30 cycles of denaturing at $95^{\circ} \mathrm{C}$ for 30 seconds, annealing at $60^{\circ} \mathrm{C}$ for 30 seconds, polymerization at $72^{\circ} \mathrm{C}$ for 30 seconds and a final 10 -min extension at $72^{\circ} \mathrm{C}$. PCR products were loaded into $2 \%$ agarose gels (Bio-Rad, CA, USA) containing $0.6 \mu \mathrm{g} / \mathrm{mL}$ ethidium bromide (Bio-Rad, CA, USA) and run in Tris-acetate-ethylenediaminetetraacetic acid buffer (Thermo Scientific, UT, USA) for $45 \mathrm{~min}$. The Alpha Innotech gel documentation station (Alpha Innotech, CA, USA) was used to observe PCR products.

\section{Statistics}

Statistical analysis was performed using analysis of variance (ANOVA) and Tukey pair-wise comparisons between each population, with a $\mathrm{p}$-value $<0.05$ being considered significant.

\section{Results}

\section{piPSC lines express pluripotent stem cell markers}

Two lines of piPSCs were derived from pig fibroblast cells by transducing with six human pluripotency genes: hPOU5F1, hNANOG, hSOX2, hLIN28, hC-MYC and hKLF4 driven by the EF1- $\alpha$ promoter. After 24 hrs, putative piPSCs were plated on feeder cells in stem cell expansion medium. piPSCs in both lines were observed as early as day 15 with colonies showing various phenotypes including those of partially and fully reprogrammed cells. Partially reprogrammed cells appeared to be granular in morphology or abnormally large with many of these cells displaying lipid droplets and loose attachment indicating cell death. Cells considered to be fully reprogrammed formed compact colonies that were manually isolated at day 25 post- transduction and were plated onto feeder cells in stem cell expansion medium (Figures $1 \mathrm{~A}$ and $1 \mathrm{~B})$. Both piPSC lines formed highly refractive colonies that at a single cell level showed clear cell borders, a high nuclear to cytoplasm ratio and large nucleoli (Figure 1C). No major morphological differences were observed between the two piPSC lines. Cells were passaged every 3 to 4 days. piPSC lines 1 and 2 were strongly positive for alkaline phosphatase (AP; Figure 1D) and immunocytochemistry results showed that both lines were positive for the pluripotent markers NANOG (Figure 1E), SOX2 (Figure 1F), POU5F1 (Figure 1G; SOX2 and POU5F1 merge Figure 1H), SSEA1 (Figure 1I), SSEA4 (Figure 1J) and TRA-1-81 (Figure $1 \mathrm{~K}$ ), while they were mostly negative for TRA-1-60 (data not shown). Pig fibroblasts (pig F) were negative for all pluripotency markers (Figure S1). Human iPSCs (hiPSCs) showed a similar expression profile to piPSC lines (Figure S2) with the exception of SSEA1 and TRA-1-60 expression, which hiPSCs were negative and positive for respectively.

\section{Activation of the pig pluripotency network in pipscs}

To determine if the 6 human reprogramming genes had integrated into the pig genome, PCR was performed using human specific primers. PCR results showed that hPOU5F1, hSOX2, hKLF4, hC-MYC and hLIN28 human reprogramming factors integrated into the genome of both piPSC lines similar to hiPSCs (Figure $2 \mathrm{~A}$ ), while the pig fibroblast parent population were negative for all 6 human genes. hNANOG was the only gene that did not successfully integrate into piPSCs. RT-PCR results showed that the 5 integrated human reprogramming genes were expressed in piPSCs similar to WA09 human ESCs (Figure 2B). Reprogramming gene expression was absent in pig fibroblast parent cells. The reverse transcription minus (RT-) control showed that there was no DNA contamination of the samples (Figure 2B). To determine if overexpression of exogenous human reprogramming genes resulted

\begin{tabular}{|l|l|l|}
\hline Primer Sequence $\mathbf{( 5}^{\prime} \mathbf{- 3}^{\prime} \mathbf{)}$ & Forward & Reverse \\
\hline hPOU5F1 & ATTTCACCAGGCCCCCGGCTT & CTTTGATGTCCTGGGACTCCTCCG \\
\hline hC-MYC & GCAGCGACTCTGAGGAGGAACAA & TTTTCCTTACGCACAAGAGTTCCGT \\
\hline hLIN28 & TCAGCCGACGACCATGGGCT & CCATGTGCAGCTTACTCTGGTGCAC \\
\hline hNANOG & TGCTGGACTGAGCTGGTTGCC & TGGAGGAAGGAAGAGGAGAGACAGT \\
\hline hKLF4 & GGCTGATGGGCAAGTTCG & CTGATCGGGCAGGAAGGAT \\
\hline hSOX2 & CCCCTGTGGTTACCTCTTCCTCC & TGCCGTTAATGGCCGTGCC \\
\hline hGAPDH & GAGTCAACGGATTTGGTCGT & TTGATTTTGGAGGGATCTCA \\
\hline pPOU5F1 & ACAAGGAGAAGCTGGAGCCG & CGCGGACCACATCCTTCTCT \\
\hline pSOX2 & CACCTACAGCATGTCCTACTCG & GGTTTTCTCCATGCTGTTTCTT \\
\hline pNANOG & TCTGTGTCAGTTTGAGGGACAGG & AACAAGTAAAGCCTCCCTATCCCA \\
\hline pLIN28 & CAGAGTAAGCTGCACATGGAGG & GTAGGCTGGCTTTCCCTTG \\
\hline pGAPDH & CTCAACGACCACTTCGTCAA & TCTGGGATGGAAACTGGAAG \\
\hline
\end{tabular}

Table 1: Primer sequences. 
Citation: Yang JY, Liu Y, Yu P, Lu Y, Hutcheson JM, et al. (2014) Culture of Pig Induced Pluripotent Stem Cells without Direct Feeder Contact in Serum Free Media. J Stem Cell Res Ther 4: 174. doi:10.4172/2157-7633.1000174

A
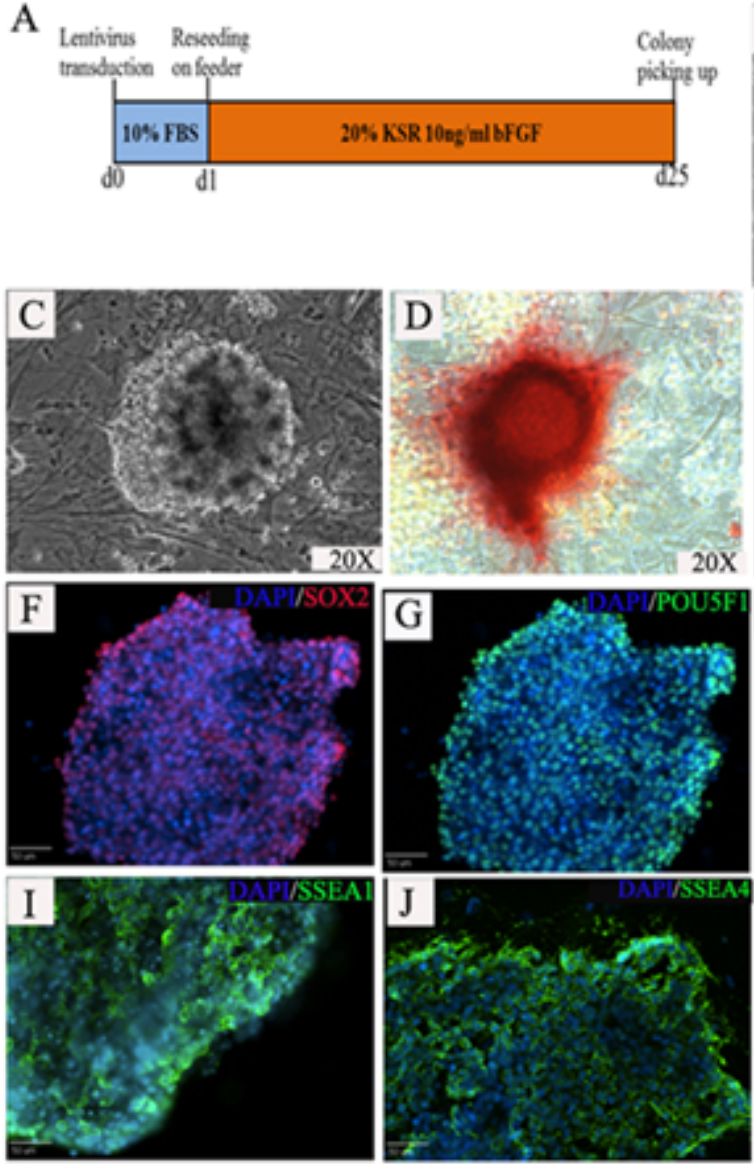
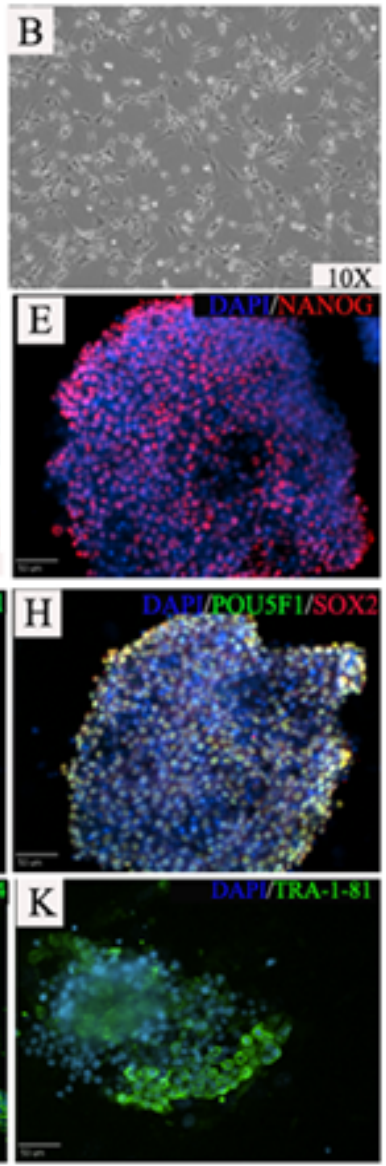

Figure 1: Generation of piPSCs from pig fibroblast cells. Approximate time table of piPSCs generation (A). Pig fibroblast showed typical flattened morphology with extensions before transduction (B). Putative piPSCs grew as small dome shaped colonies showing well defined borders at day 15 post-transduction with single cells displaying large nucleoli and high nuclear to cytoplasm ratios typical of iPSC morphology (C). piPSCs stained positive for alkaline phosphatase (D). Immunostaining demonstrated that piPSCs were strongly positive for the pluripotent factors NANOG (E); Dapi nuclear marker shown in blue), SOX2 (F) and POU5F1 (G; H-POU5F1 and SOX2 merge). piPSCs were also positive for the stem cell specific surface antigens SSEA1 (I), SSEA4 (J) and TRA-1-81 (K).
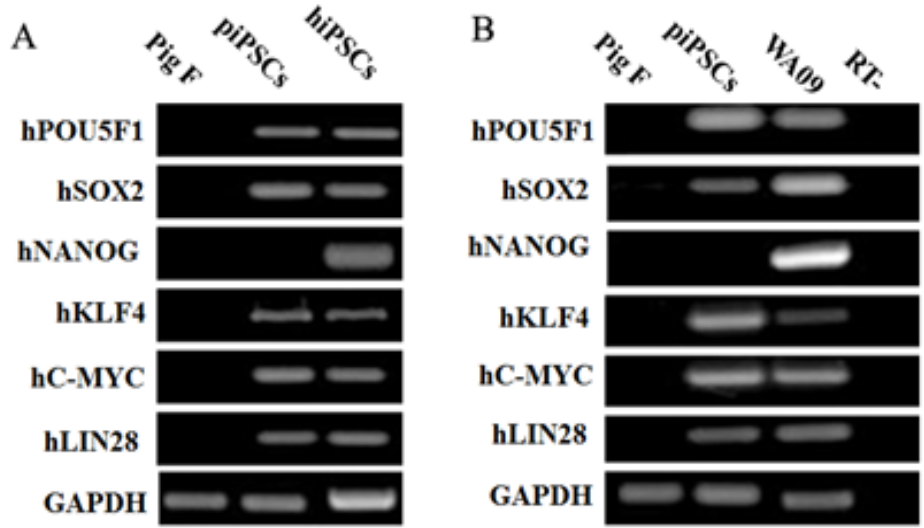

C

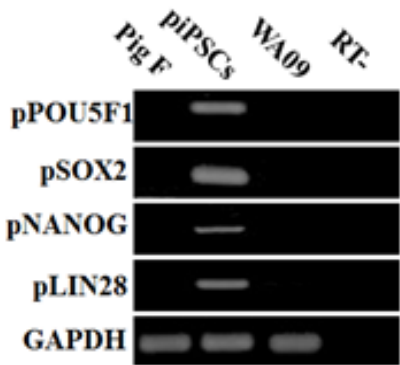

Figure 2: Activation of the pig pluripotency network in piPSCs. PCR using human specific primers demonstrated that lentivirus delivered hPOU5F1, hSOX2, hKLF4, hC-MYC and hLIN28 human reprogramming genes were integrated into the genome of piPSCs similar to positive control hiPSCs derived using the same factors (A). Pig fibroblasts (Pig F) were negative for all 6 human genes. RT-PCR results showed that the 5 lentivirus delivered and integrated human reprogramming genes were expressed at the mRNA level in piPSCs as well as WA09 hESCs positive control cells, while pig fibroblasts were negative (B). RT negative (RT-) controls showed that there was no DNA contamination of the samples. RT-PCR with pig specific primers showed that porcine pPOU5F1, pSOX2, pNANOG and pLIN28 genes were expressed in piPSCs and therefore activation of the endogenous pig pluripotency network (C). WA09 hESC and pig fibroblasts were negative for the expression of pig specific gene expression (C). 
in the activation of endogenous pig genes, RT-PCR was performed with pig endogenous specific primers. RT-PCR results showed that the porcine POU5F1, SOX2, NANOG and LIN28 genes were reactivated, while WA09 hESCs and pig fibroblast cells were negative for the expression of these genes (Figure 2C). These results indicate the integration and expression of the exogenous genes and the reactivation of the endogenous pluripotency network.

\section{Highly proliferative piPSC lines maintain a normal karyotype over extended passages}

Pluripotent stem cells characteristically demonstrate high levels of telomerase activity and rapid proliferation rates. Telomerase activity, reported in units of total product generated (TPG), was significantly ( $\mathrm{p}$-value $<0.01)$ increased in piPSC line 1 (335.1 TPG) and piPSC line 2 (261.5 TPG) relative to the pig fibroblast parent population (47.6 TPG; Figure $3 \mathrm{~A})$. piPSC cell line 1 showed telomerase activity comparable to WA09 hESCs (356 TPG), but lower than the HeLa cell line control (465 TPG). The doubling times of piPSC cell lines were determined by quantification of cell counts every 12 hours for 48 hours. The population doubling times of piPSC line 1 and 2 were 19.2 and $20 \mathrm{hrs}$ respectively, which were significantly ( $\mathrm{p}$-value $<0.01$ ) faster than the pig fibroblast parent cell line $(37.5 \mathrm{hr})$ and similar to WA09 hESC (22.7 hr) (Figure 3B). piPSC line 1 and 2 possessed a normal karyotype even after 20 passages (Figure 3C).

\section{High levels of SSEA1+ and SSEA4+ piPSCs maintained in feeder free conditions}

Typically, piPSCs have been maintained on feeder cells in media containing serum which could potentially expose piPSCs to contaminating factors (e.g. prions, viruses) or lead to the exchange of genetic information between mouse and pig cells [25]. Therefore, it is preferable to use a feeder free system such as the mTeSR1 (mTeSR1 [29]) or $2 \mathrm{i}+\mathrm{LIF}$ (NCP [27]) system. To determine the potential of mTeSR 1 or $2 \mathrm{i}+\mathrm{LIF}$ systems to maintain piPSCs in a pluripotent state, these two culture systems were compared to various DMEM/F12 and conditioned (exposed to feeder cells for 24 hours) KSR (cKSR) based media systems. None of these systems contained serum. Live stained SSEA $1+$ cells from line 1 were manually passaged onto Matrigel in one of 7 different media types: 1. mTeSR1 (mTeSR1 only); 2. KSRF (cKSR+10 ng/ml FGF2); 3. KSRFL (cKSR+10 ng/ml FGF2+10 ng/ $\mathrm{ml} \mathrm{LIF}) ; 4$. KFC (cKSR+10 ng/ml FGF2+3 uM CHIR99021); 5. KFP
(cKSR+10 ng/ml FGF2+0.8 uM PD0325901); 6. KFCP (cKSR+10 ng/ ml FGF2+3 uM CHIR99021+0.8 uM PD0325901); 7. NCP (N2B27+10 $\mathrm{ng} / \mathrm{ml} \mathrm{LIF+3} \mathrm{uM} \mathrm{CHIR99021+0.8} \mathrm{uM} \mathrm{PD0325901).} \mathrm{These} \mathrm{cells} \mathrm{were}$ then examined for pluripotency marker expression after 6 passages. Immunocytochemistry revealed that piPSCs were strongly positive for the pluripotent factors POU5F1 (Figure 4A) and NANOG (Figure 4B) in all conditions. piPSCs expanded in all 7 conditions were also positive for stem cell specific surface antigens SSEA1 (Figure 4C), SSEA4 (Figure 4D) and TRA-1-81 (Figure 4F), although fewer cells were positive for these markers relative to POU5F1 and NANOG. Only a small subset of cells in the 7 conditions were TRA-1-60 positive (Figure 4E). To quantitatively determine the optimum culture condition, flow cytometry was performed on each cell population for SSEA1 and SSEA4 stem cell markers. A large percentage of cells were SSEA1+ (Figure 4G) and SSEA4+ (Figure 4H) in conditions KSRF, KSRFL and KFP with $>75 \%$ of the cells being SSEA $1+$ and $>20 \%$ being SSEA $4+$. Conditions mTeSR1, KFC, KFCP and NCP showed significantly lower levels of SSEA1+ cells and conditions mTeSR1, KFC and KFCP showed significantly lower SSEA4+ cells. These results demonstrate that piPSCs can be propagated in a feeder free system; however, feeder conditioned media resulted in more SSEA1 and SSEA4 positive piPSCs than nonfeeder conditioned systems (mTeSR1 and NCP).

\section{Pig iPSCs differentiate into all three germ layers during embryoid body differentiation}

The developmental plasticity of piPSC lines were tested by EB differentiation and immunocytochemistry analysis for cells representing all 3 germlayers. Cells underwent 8 days of EB differentiation (Figure $5 \mathrm{~A})$. EBs was replated for an additional 2 days in $20 \%$ KSR medium without FGF2. Immunocytochemistry results showed cells from plated EBs were positive for $\beta$ III-TUBULIN (ectoderm, Figure 5B), aSMA (mesoderm, Figure 5C) and Vimentin (endoderm, Figure 5D). These results indicated that piPSCs differentiated into cell types from all three germ layers in vitro.

\section{Discussion}

piPSCs derived in this study displayed immunoreactivity and morphology similar to mouse [19,30,31], human [18,32,33] and previously derived porcine $[20,21]$ iPSCs, consistent with pluripotency. piPSCs reprogrammed from pig fibroblast cells using 6 human reprogramming genes showed typical stem cell morphology and
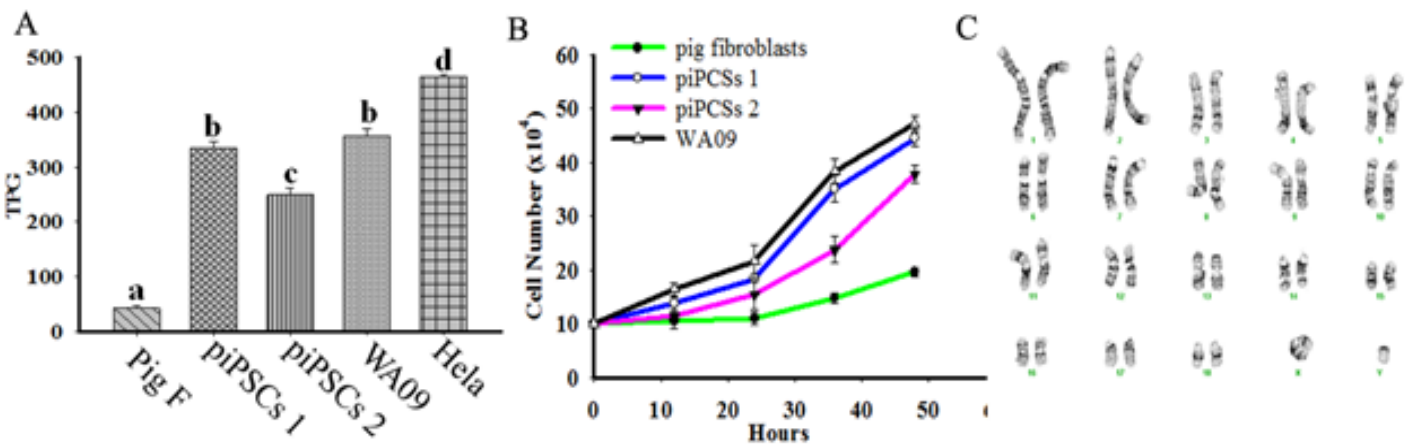

Figure 3: piPSCs demonstrate high telomerase activity and rapid proliferation. Telomerase activities in piPSC line 1 and 2 were significantly ( $p$-value<0.01) higher than pig fibroblast cells (A; telomerase levels reported in units of total product generated (TPG)). piPSC line 1 showed comparable telomerase activity to WA09 hESCs control. Telomerase activity of piPSC line 1 and 2 were lower than the HeLa cell control. piPSC line 1 and 2 doubling times were 19.2 and 20 hrs respectively, which were significantly ( $p$-value<0.01) faster than the pig fibroblast cells ( $37.5 \mathrm{hrs})$ and similar to the WA09 hESCs $(22.7 \mathrm{hrs}$; B). piPSC lines showed a normal karyotype after 20 passages (C). Bars which are not denoted by a common letter (a-d) show a statistically significant difference, $p$-value $<0.05$. 
Citation: Yang JY, Liu Y, Yu P, Lu Y, Hutcheson JM, et al. (2014) Culture of Pig Induced Pluripotent Stem Cells without Direct Feeder Contact in Serum Free Media. J Stem Cell Res Ther 4: 174. doi:10.4172/2157-7633.1000174
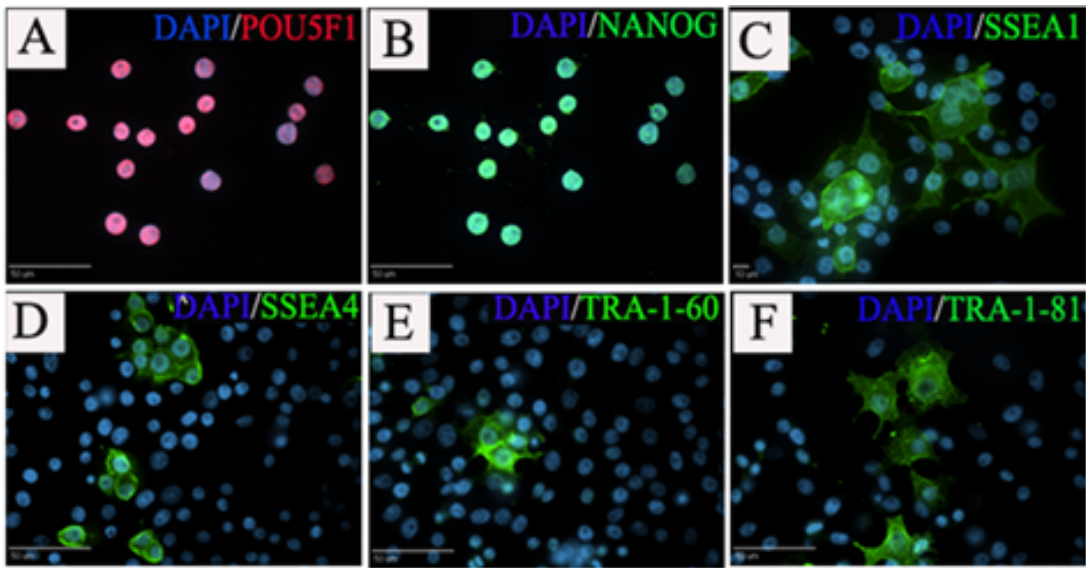

G

$\mathrm{H}$
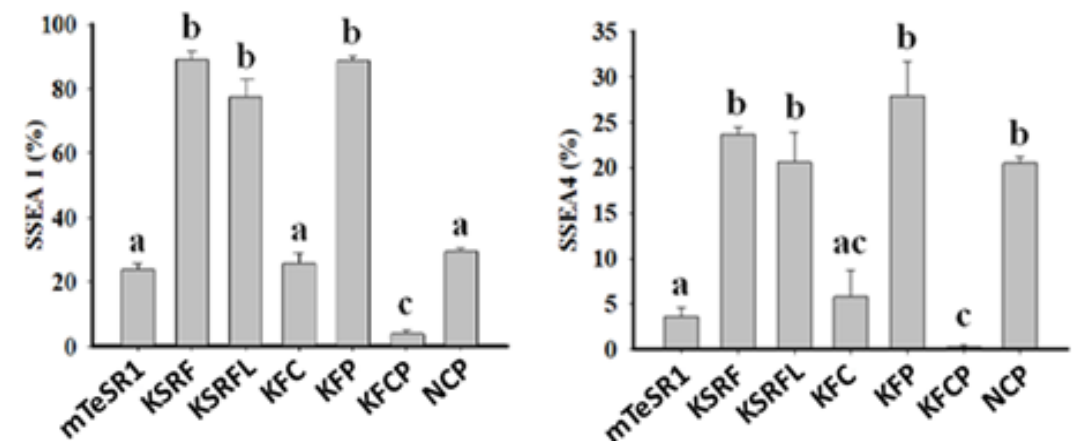

Figure 4: Culture of piPSC in feeder free conditions. Live stained SSEA1+ piPSCs were manually passaged onto Matrigel in one of 7 different media types: mTeSR1, KSRF, KSRFL, KFC, KFP, KFCP and NCP. After 6 passages, immunocytochemistry results showed that piPSCs were strongly positive for the introduced factors POU5F1(A; cells in mTeSR1 shown) and NANOG (B) and non-overexpressed stem cell markers SSEA1(C), SSEA4 (D), TRA-1-60 (E) and TRA-1-81 (F) in all conditions. Flow cytometry analysis of cells at passage 6 showed that $>75 \%$ of cells were SSEA1+ (G) and $>20 \%$ being SSEA4+ $(\mathrm{H})$ in $\mathrm{KSRF}$, KSRFL and KFP conditions. mTeSR1, KFC, KFCP and NCP conditions showed significantly lower levels of SSEA1+ cells and mTeSR1, KFC, KFCP conditions showed significantly lower SSEA4+ cells. Bars which are not denoted by a common letter (a-d) show a statistically significant difference, $p$-value $<0.05$.
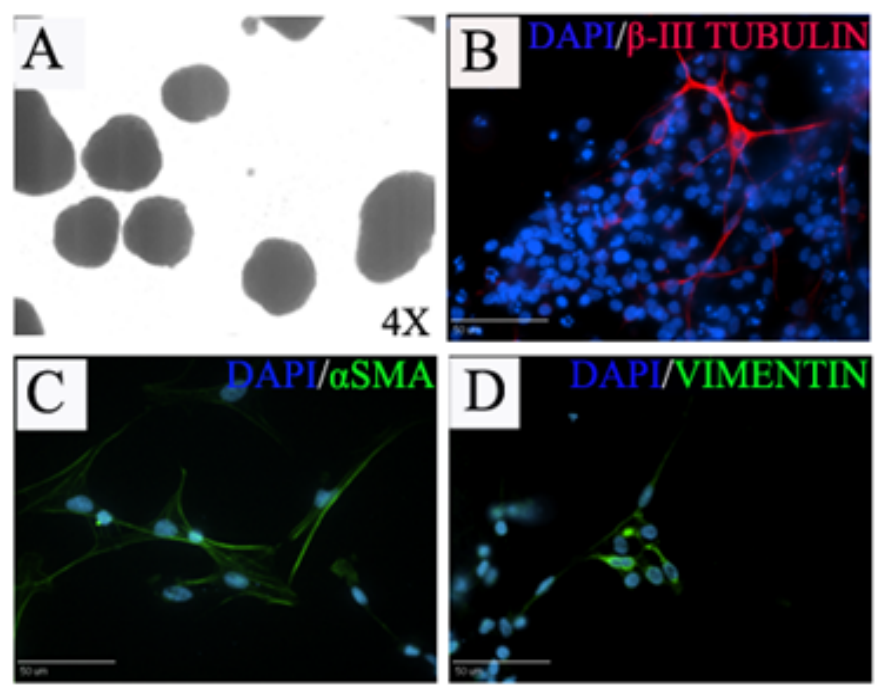

Figure 5: Differentiation of piPSCs into cells representing all 3 germ layers. piPSCs formed compacted EBs (A) and underwent 10 days of EB differentiation. Immunostaining of plated EBs showed that cells were positive for the ectoderm marker BIII-TUB (B), mesoderm marker aSMA (C) and endoderm marker Vimentin (D). Scale bars=50um. 
displayed a high nuclear to cytoplasm ratio with large nucleoli. These cells were highly positive for stem cell markers AP, POU5F1, SOX2, NANOG, SSEA1, SSEA4 and TRA-1-81. piPSCs also showed similar expansion patterns and proliferation rates to hESCs and were able to maintain karyotypic stability in extended culture. These cells were then tested for their potential to be supported in serum and feeder free conditions.

Feeder-free maintenance of pig pluripotent stem cells has significant technical and scientific advantages over the use of non-defined systems. Recent developments in pluripotent stem cell culture have led to new systems for maintaining human and mouse pluripotent stem cells in both feeder and serum free conditions. Past attempts at adapting these human and mouse stem cell culture systems to pig cells have been met with significant challenges. When used to expand pig ESCs, established feeder and serum based human and mouse cell culture systems resulted in slow growth rates, spontaneous differentiation, degeneration and a gradual decline in the number of surviving cells within a limited number of passages [15,34-37]. This suggests that there are important species specific differences. Species specific differences can even be observed between mouse and human feeder free systems, with the mouse system being LIF dependent and the human system being FGF2 dependent $[38,39]$.

With respect to pig iPSCs, previous groups reported successful generation of piPSCs which were FGF2 dependent [20,21], FGF2 independent [40] and both FGF2 and LIF dependent [41]. To further elucidate the optimum culture conditions for piPSCs, we compared the established mouse pluripotent stem cell $2 \mathrm{i}+\mathrm{LIF}$ system (NCP), the human pluripotent stem cell mTeSR1 system (mTeSR1), the human pluripotent stem cell feeder conditioned media system (KSRF) and variations of these systems; none of which contain serum $[26,42,43]$. mTeSR1 has previously been shown to support the maintenance of piPSCs, which was confirmed by these findings [16]. However, treatments where feeder conditioned media were utilized resulted in higher levels of SSEA1 and SSEA4 positive populations relative to the mTeSR1 and 2i+LIF system. The high percentage of SSEA1 and SSEA4 positive populations in feeder conditioned media culture systems clearly demonstrated that feeder cells produce factors, or concentrations of factors, that are important for maintaining pluripotency expression in piPSCs. In a previous study, the extracellular signal-regulated kinase (ERK) pathway inhibitor PD0325901 (PD) and glycogen synthase kinase-3beta (GSK-3B) pathway inhibitor CHIR99021 (CH) were utilized in the development of piPSCs and led to increased colony compaction and proliferation [20]. However, expansion of piPSCs in PD0325901 and CHIR99021 or CHIR99021 without PD032591 led to significant reductions in SSEA1 and SSEA4 positive cell populations in this study. The increased numbers of SSEA1 and SSEA4 negative cells in these conditions indicate that CHIR99021 may cause increased differentiation of piPSCs. Previous studies of GSK-3 inhibition in human pluripotent stem cells have shown increased differentiation in contrast to mouse pluripotent stem cells $[44,45]$. Based on this evidence, piPSCs may respond more similarly to human than mouse cells in terms of inhibition of the GSK-3 pathway. These results demonstrate that piPSCs can be cultured in feeder and serum free systems, yet feeder derived factors combined with FGF2 may be needed to maintain pluripotency and prevent differentiation long term.

Previous studies have also shown inconsistencies in the expression of pluripotent stem cell markers in piPSCs. The best markers to test are those that are not over expressed, as they are not confounded by exogenous genes, yet show significant divergence in expression.
Previous reports showed piPSCs were weakly positive or negative for SSEA4 and TRA-1-81[16,21], while others showed piPSCs were positive for both SSEA4 and TRA-1-81[20,22]. piPSCs in this study were positive for SSEA4 and TRA1-81. Additionally, they were strongly positive for SSEA1, which is expressed in mouse pluripotent stem cells and not human [19]. Since mouse and human pluripotent stem cells have distinct SSEA1 and SSEA4 marker expression, it is not surprising that porcine cells may differ. Additional studies are needed to further identify the specific immunoreactivity signature that results in a truly pluripotent stem cell population.

In conclusion, piPSCs offer a unique potential to genetically manipulate pigs for improved utility in both agriculture and the biomedical sciences. The potential use of iPSCs in these fields, especially in the context of food production or xenotransplantation, highlights the importance of developing maintenance systems that are free of potential contaminants. Here we demonstrated that piPSCs could be maintained over multiple passages without direct feeder contact in serum free media. The culture conditions for piPSCs developed in this report advance the field closer to a completely xeno-free expansion system, ultimately expanding the potential and utility of these cells for biomedical and agricultural applications.

\section{Acknowledgements}

We would like to thank Mrs. Julie Nelson and the Center for Tropical and Emerging Global Diseases Flow Cytometry Facility, University of Georgia. We would also like to thank Mike Daniel, Amber Williams, the Large Animal Research Unit and the Double Bridges Swine Unit for their assistance with sample collection.

\section{References}

1. Ekser B, Ezzelarab M, Hara H, van der Windt DJ, Wijkstrom M, et al. (2012) Clinical xenotransplantation: the next medical revolution? Lancet 379: 672-683.

2. Rogers CS, Stoltz DA, Meyerholz DK, Ostedgaard LS, Rokhlina T, et al. (2008) Disruption of the CFTR gene produces a model of cystic fibrosis in newborn pigs. Science 321: 1837-1841.

3. Xi S, Yin W, Wang Z, Kusunoki M, Lian X, et al. (2004) A minipig model of highfat/high-sucrose diet-induced diabetes and atherosclerosis. Int J Exp Patho 85: 223-231.

4. Ali A, Patel A, Ali Z, Abu-Omar Y, Freed D, et al. (2010) Medium to long-term clinical outcome following stentless aortic valve replacement: comparison between allograft and xenograft valves. Interact Cardiovasc Thorac Surg 11 166-170.

5. Lai L, Kang JX, Li R, Wang J, Witt WT, et al. (2006) Generation of cloned transgenic pigs rich in omega-3 fatty acids. Nat Biotechnol 24: 435-436.

6. Lai L, Kolber-Simonds D, Park KW, Cheong HT, Greenstein JL, et al. (2002) Production of alpha-1,3-galactosyltransferase knockout pigs by nuclear transfer cloning. Science 295: 1089-1092.

7. Evans MJ, Kaufman MH (1981) Establishment in culture of pluripotential cells from mouse embryos. Nature 292: 154-156.

8. Martin GR (1981) Isolation of a pluripotent cell line from early mouse embryos cultured in medium conditioned by teratocarcinoma stem cells. Proc Natl Acad Sci USA 78: 7634-7638.

9. Masuya H, Nishida K, Furuichi T, Toki H, Nishimura G, et al. (2007) A nove dominant-negative mutation in Gdf5 generated by ENU mutagenesis impairs joint formation and causes osteoarthritis in mice. Hum Mol Genet 16: 23662375 .

10. Aigner B, Rathkolb B, Herbach N, Hrabé de Angelis M, Wanke R, et al. (2008) Diabetes models by screen for hyperglycemia in phenotype-driven ENU mouse mutagenesis projects. Am J Physiol Endocrinol Metab 294: E232-240.

11. Bühling F, Kouadio M, Chwieralski CE, Kern U, Hohlfeld JM, et al. (2011) Gene targeting of the cysteine peptidase cathepsin $\mathrm{H}$ impairs lung surfactant in mice. PLoS One 6: e26247.

12. Muller $U$ (1999) Ten years of gene targeting: targeted mouse mutants, from vector design to phenotype analysis. Mech Dev 82: 3-21. 
Citation: Yang JY, Liu Y, Yu P, Lu Y, Hutcheson JM, et al. (2014) Culture of Pig Induced Pluripotent Stem Cells without Direct Feeder Contact in Serum Free Media. J Stem Cell Res Ther 4: 174. doi:10.4172/2157-7633.1000174

Page 8 of 8

13. Strojek RM, Reed MA, Hoover JL, Wagner TE (1990) A method for cultivating morphologically undifferentiated embryonic stem cells from porcine blastocysts. Theriogenology 33: 901-913.

14. Li M, Zhang D, Hou Y, Jiao L, Zheng X, et al. (2003) Isolation and culture of embryonic stem cells from porcine blastocysts. Mol Reprod Dev 65: 429-434.

15. Piedrahita JA, Anderson GB, Bondurant RH (1990) On the isolation of embryonic stem cells: Comparative behavior of murine, porcine and ovine embryos. Theriogenology 34: 879-901.

16. West FD, Terlouw SL, Kwon DJ, Mumaw JL, Dhara SK, et al. (2010) Porcine induced pluripotent stem cells produce chimeric offspring. Stem Cells Dev 19: 1211-1220.

17. West FD, Uhl EW, Liu Y, Stowe H, Lu Y, et al. (2011) Chimeric Pigs Produced from Induced Pluripotent Stem Cells Demonstrate Germline Transmission and No Evidence of Tumor Formation in Young Pigs. Stem Cells 29: 1640-1643.

18. Takahashi K, Tanabe K, Ohnuki M, Narita M, Ichisaka T, et al. (2007) Induction of pluripotent stem cells from adult human fibroblasts by defined factors. Cell 131: 861-872.

19. Takahashi K, Yamanaka S (2006) Induction of pluripotent stem cells from mouse embryonic and adult fibroblast cultures by defined factors. Cell 126: 663-676.

20. Esteban MA, Xu J, Yang J, Peng M, Qin D, et al. (2009) Generation of induced pluripotent stem cell lines from Tibetan miniature pig. J Biol Chem 284: 1763417640

21. Ezashi T, Telugu BP, Alexenko AP, Sachdev S, Sinha S, et al. (2009) Derivation of induced pluripotent stem cells from pig somatic cells. Proc Natl Acad Sci USA 106: 10993-10998.

22. Wu Z, Chen J, Ren J, Bao L, Liao J, et al. (2009) Generation of Pig-Induced Pluripotent Stem Cells with a Drug-Inducible System. J Mol Cell Biol 1: 46-54.

23. Liu Y, Yang JY, Lu Y, Yu P, Dove CR, et al. (2013) alpha-1,3Galactosyltransferase Knockout Pig Induced Pluripotent Stem Cells: A Cell Source for the Production of Xenotransplant Pigs. Cell Reprogram 15: 107-116.

24. Chaudhry MA, Vitalis TZ, Bowen BD, Piret JM (2008) Basal medium composition and serum or serum replacement concentration influences on the maintenance of murine embryonic stem cells. Cytotechnology 58: 173-179.

25. Martin MJ, Muotri A, Gage F, Varki A (2005) Human embryonic stem cells express an immunogenic nonhuman sialic acid. Nat Med 11: 228-232.

26. Ying QL, Wray J, Nichols J, Batlle-Morera L, Doble B, et al. (2008) The ground state of embryonic stem cell self-renewal. Nature 453: 519-523

27. Silva J, Barrandon O, Nichols J, Kawaguchi J, Theunissen TW, et al. (2008) Promotion of reprogramming to ground state pluripotency by signal inhibition. PLoS Biol 6: e253.

28. West FD, Mumaw JL, Gallegos-Cardenas A, Young A, Stice SL (2011) Human Haploid Cells Differentiated from Meiotic Competent Clonal Germ Cell Lines that Originated from Embryonic Stem Cells. Stem Cells Dev 20: 1079-1088.

29. Ludwig TE, Bergendahl V, Levenstein ME, Yu J, Probasco MD, et al. (2006) Feeder-independent culture of human embryonic stem cells. Nat Methods 3 : 637-646.
30. Wernig M, Meissner A, Foreman R, Brambrink T, Ku M, et al. (2007) In vitro reprogramming of fibroblasts into a pluripotent ES-cell-like state. Nature 448 318-324.

31. Okita K, Ichisaka T, Yamanaka S (2007) Generation of germline-competent induced pluripotent stem cells. Nature 448: 313-317.

32. Yu J, Vodyanik MA, Smuga-Otto K, Antosiewicz-Bourget J, Frane JL, et al (2007) Induced pluripotent stem cell lines derived from human somatic cells. Science 318: 1917-1920.

33. Park IH, Zhao R, West JA, Yabuuchi A, Huo H, et al. (2008) Reprogramming of human somatic cells to pluripotency with defined factors. Nature 451: 141-146.

34. Notarianni E, Laurie S, Moor RM, Evans MJ (1990) Maintenance and differentiation in culture of pluripotential embryonic cell lines from pig blastocysts. J Reprod Fertil Suppl 41: 51-56.

35. Talbot NC, Rexroad CE Jr, Pursel VG, Powell AM, Nel ND (1993) Culturing the epiblast cells of the pig blastocyst. In Vitro Cell Dev Biol Anim 29: 543-54.

36. Vackova I, Novakova Z, Krylov V, Okada K, Kott T, et al. (2011) Analysis of marker expression in porcine cell lines derived from blastocysts produced in vitro and in vivo. J Reprod Dev 57: 594-603.

37. du Puy L, Lopes SM, Haagsman HP, Roelen BA (2011) Analysis of coexpression of OCT4, NANOG and SOX2 in pluripotent cells of the porcine embryo, in vivo and in vitro. Theriogenology 75: 513-526

38. Hall J, Guo G, Wray J, Eyres I, Nichols J, et al. (2009) Oct4 and LIF/Stat3 additively induce Kruppel factors to sustain embryonic stem cell self-renewal. Cell Stem Cell 5: 597-609.

39. Vallier L, Alexander M, Pedersen RA (2005) Activin/Nodal and FGF pathways cooperate to maintain pluripotency of human embryonic stem cells. J Cell Sci 118: $4495-4509$.

40. Wu Z, Chen J, Ren J, Bao L, Liao J, et al. (2009) Generation of pig induced pluripotent stem cells with a drug-inducible system. J Mol Cell Biol 1: 46-54.

41. Telugu BP, Ezashi T, Roberts RM (2010) Porcine induced pluripotent stem cells analogous to naive and primed embryonic stem cells of the mouse. Int $J$ Dev Biol 54: 1703-1711.

42. Ludwig TE, Levenstein ME, Jones JM, Berggren WT, Mitchen ER, et al. (2006) Derivation of human embryonic stem cells in defined conditions. Nat Biotechnol 24: 185-187.

43. Xu C, Inokuma MS, Denham J, Golds K, Kundu P, et al. (2001) Feeder-free growth of undifferentiated human embryonic stem cells. Nat Biotechnol 19 971-974.

44. Bone HK, Nelson AS, Goldring CE, Tosh D, Welham MJ (2011) A nove chemically directed route for the generation of definitive endoderm from human embryonic stem cells based on inhibition of GSK-3. J Cell Sci 124: 1992-2000.

45. Lian X, Hsiao C, Wilson G, Zhu K, Hazeltine LB, et al. (2012) Robust cardiomyocyte differentiation from human pluripotent stem cells via temporal modulation of canonical Wnt signaling. Proc Natl Acad Sci U S A 109: E18481857. 